\title{
Systematic Review and Meta-Analysis of Inflammatory Bowel Disease Adverse Events with Anti-Interleukin 17A Agents and Tumor Necrosis Factor Inhibitors in Rheumatic Disease and Skin Psoriasis
}

\author{
Steven L. Truong · Jasmine Chin · David F. L. Liew · Syeda Farah Zahir • \\ Elizabeth G. Ryan · Diana Rubel · Graham Radford-Smith • \\ Philip C. Robinson (D)
}

Received: July 17, 2021 / Accepted: August 11, 2021 / Published online: August 26, 2021

(c) The Author(s) 2021

\begin{abstract}
Introduction: The aim of this work is to perform a systematic review and meta-analysis of anti-tumor necrosis factor (anti-TNF) and antiinterleukin-17 (anti-IL-17) trials for spondyloarthritis, psoriatic arthritis, and psoriasis comparing rates of inflammatory bowel disease (IBD) events compared to placebo.
\end{abstract}

Supplementary Information The online version contains supplementary material available at https:// doi.org/10.1007/s40744-021-00360-6.

S. L. Truong

Department of Medicine, Griffith University, Brisbane, QLD, Australia

\section{S. L. Truong}

Coast Joint Care, Maroochydore, QLD, Australia

J. Chin · E. G. Ryan · G. Radford-Smith ·

P. C. Robinson

Faculty of Medicine, University of Queensland,

Brisbane, QLD, Australia

D. F. L. Liew

Department of Medicine, University of Melbourne,

Parkville, Australia

D. F. L. Liew

Department of Clinical Pharmacology and

Therapeutics, Austin Health, Heidelberg, VIC, Australia

D. F. L. Liew

Department of Rheumatology, Austin Health,

Heidelberg, VIC, Australia
Methods: MEDLINE, EMBASE, and The Cochrane Library were searched for doubleblind, randomized placebo-controlled anti-TNF and anti-IL-17 trials of included diseases. Inflammatory bowel disease events from the RCT period were pooled and meta-analyzed using statistical methods suitable for low-eventrate meta-analysis (Peto's, Mantel-Haenszel, hypergeometric-normal model, and ShusterGuo-Skyler). When observed data were insufficient, we performed an exploratory sensitivity analysis to compare methods.

\section{S. F. Zahir · E. G. Ryan}

QCIF Facility for Advanced Bioinformatics, Institute for Molecular Bioscience, The University of Queensland, Brisbane, QLD, Australia

\section{Rubel}

Australian National University and Woden

Dermatology, Canberra, Australia

\section{P. C. Robinson}

Royal Brisbane and Women's Hospital, Metro North Hospital and Health Service, Brisbane, QLD, Australia

P. C. Robinson ( $₫)$

School of Clinical Medicine, University of Queensland, Royal Brisbane and Women's Hospital, Bowen Bridge Road, Herston, QLD 4006, Australia e-mail: philip.robinson@uq.edu.au 
Results: We identified 9551 original papers, and included 96 publications: 65 anti-TNF and 31 anti-IL-17 trials, containing 21 new and 12 flare IBD events in 28,209 participants. New IBD on anti-IL-17 occurred $0.23 / 100$ patient-years (PY) in psoriasis, 0.61/100 PY in PsA and 1.63/ $100 \mathrm{PY}$ in spondyloarthritis, rates similar to observational cohorts, and less commonly on anti-TNF (0/100 PY, 0/100 PY, 0.32/100 PY, respectively). No evidence of difference between groups was found, with wide CI from many pooled counts of zero, especially in placebo arms.

Conclusions: IBD events were rare, occurring at rates similar to biologic-naive groups. We could not find statistically significant differences in risk of new or recurrent IBD between treatment and control groups using selected meta-analytical methods for low event rate scenarios. Metaanalyses of this topic require more IBD events, ideally without pooling heterogeneous groups. Larger, thoroughly reported trials with systematic and detailed safety reporting are required to improve risk estimation and to make accurate inferences.

Keywords: Inflammatory bowel disease; Antiinterleukin-17; Anti-tumor necrosis factor; Axial spondyloarthritis; Psoriatic arthritis; Psoriasis; Meta-analysis; Crohn's disease; Ulcerative colitis; Pharmacovigilance

\section{Key Summary Points}

Why carry out this study?

Anti-IL-17A trials for psoriasis, psoriatic arthritis, and spondyloarthritis have reported IBD events but no causative link or increased risk has been formally identified so far.

The study used a systematic review and meta-analysis methodology to try to determine if anti-IL-17 agents and antiTNF agents were associated with inflammatory disease adverse events in those with psoriasis, spondylarthritis, and psoriatic arthritis.

\section{What was learned from the study?}

Pooled data from blinded RCTs of these three diseases finds IBD events to be very rare.

The low event rate of inflammatory bowel disease adverse events makes it challenging to determine whether there is a statistically significant increased rate.

Recognized methods of hypothesis testing these rare events have limitations and produce wide confidence intervals.

\section{INTRODUCTION}

Axial spondyloarthritis, psoriasis, and psoriatic arthritis are immune-mediated diseases which share genetic risk loci, an increased prevalence of inflammatory bowel disease (IBD), and can be treated with anti-tumor necrosis factor-alpha (anti-TNF) and anti-interleukin-17 (anti-IL-17) agents [1, 2]. IL-17 has multiple known functions including anti-fungal immunity, mucosal inflammation, and tissue repair. It is produced in response to IL-23 in several diseases and may be produced through other mechanisms [3, 4]. It has complex regulatory actions, promoting the inflammatory effects of T helper 17 effector cells over regulatory $\mathrm{T}$ cells and has a self-inhibitory negative feedback loop [5-7]. In IBD, IL-17 is elevated in serum and the intestinal mucosa, but its blockade was not effective in two human trials of Crohn's disease, where a possible increase in disease activity was observed [8-10].

Therapy in IBD and spondyloarthritis uncommonly causes paradoxical effects, including anti-TNF induced psoriasis and a range of demyelinating disorders [11-13]. Additionally, there is an increased background risk of IBD in patients with axial spondyloarthritis, psoriatic arthritis, and to a lesser extent skin psoriasis, independent of treatment initiation [14]. On this background, incident IBD events have developed in randomized controlled trials (RCTs) and post-RCT followups of anti-IL-17 agents for spondyloarthritis, 
skin psoriasis, and psoriatic arthritis. This raises the question of whether exposure to anti-IL-17A agents promotes IBD pathogenesis or whether IBD events represent background risk in this atrisk population.

The frequency of IBD events in these trials is uncommon, limiting the use of commonly used statistical techniques [15]. Single medication integrated pooled analyses of IL-17 inhibitors secukinumab or ixekizumab did not demonstrate elevated rates of IBD compared to the disease population, while two meta-analyses found no risk difference; however, we believe that each of these approaches has methodological limitations [16-22].

Here, we present a systematic literature review and meta-analysis that collates the most up-to-date clinical trial data comparing new and flare IBD events in placebo-controlled antiTNF and anti-IL-17 RCTs. It captures more IBD events than its predecessors and was designed to minimize bias and to examine four methods of low event rate meta-analysis to explore appropriate techniques in this scenario.

\section{METHODS}

\section{Search Strategy and Study Selection Criteria}

We searched for published RCTs in MEDLINE, EMBASE, and the Cochrane Library from inception until October 1, 2019. Search terms validated to find RCTs in MEDLINE and EMBASE were used (Supplementary Material). Bibliographical references of individual publications that met inclusion criteria were checked to identify other relevant studies. The review was not registered. The protocol, template data collection forms, data extracted from included studies, data used for all analyses, analytic code, and any other materials used in the review are available from the corresponding author. No amendments were made to the protocol after finalization.

Inclusion was based on predefined criteria and involved study title, abstract, and full text review in a hierarchical manner. Two authors (ST, JC) carried out independent screening and disagreements were resolved by discussion. Studies were considered eligible if they were RCTs in psoriatic arthritis, psoriasis or ankylosing spondylitis, non-radiographic axial spondyloarthritis, or peripheral spondyloarthritis (the last three grouped as spondyloarthritis). Included interventions were anti-IL17 or anti-TNF agents with placebo as comparator. Outcomes were flares of known IBD or new-onset IBD, defined by each study's protocol.

Only safety data from the double-blinded period of each trial were used, and only from trials with placebo-controlled double-blinded biologic arms.

Studies were excluded if they involved children, had insufficient adverse event reporting, were not in English, less than 8 weeks in duration, or were secondary publications of included trials.

After removal of duplicates, publications were screened by title and/or abstract then excluded or progressed to full-text review. The supplementary appendices of published papers and/or relevant clinical trials database were examined if full-text review was insufficient to assess exclusion criteria and results. Trial sponsors were contacted to clarify IBD events that were inadequately described in the primary manuscript.

For each publication, two reviewers (JC, ST) independently extracted the following information to a spreadsheet: publication identifiers, disease, ethnicity, age, gender, whether previous or active IBD was excluded, primary outcome(s), interventions, comparator, intervention arm sizes, duration of doubleblinded period, new IBD cases per arm, flares of known IBD per arm, and number of subjects with known IBD per arm. We combined multiple intervention dose arms into a single treatment arm.

Study quality was assessed by two independent reviewers using Jadad scoring [23]. Quality assessment was conducted in the areas of adequate sequence generation, allocation of concealment, blinding and follow-up/drop out. Review of supplementary materials and the relevant clinical trials database was done if the 
publication lacked relevant detail (Supplementary Material).

We performed separate meta-analyses of new IBD and IBD flares for participants with skin psoriasis, psoriatic arthritis, and spondyloarthritis (combined axial spondyloarthritis and peripheral spondyloarthritis), where trials defined the cohort in this way for example [24]. We did not pool new IBD events with flares of known disease, as these acute and chronic inflammatory events may not share pathological triggers and the trials of anti-IL-17 for Crohn's disease did not definitively demonstrate more flares on treatment.

Anticipating low event rates, we explored four meta-analysis methods recommended for low event rates: Peto's one-step, Mantel-Haenszel $(\mathrm{MH})$, hypergeometric-normal model (HG) and Shuster, Guo and Skyler (SGS) [25-28]. We conducted and compared fixed-effect (Peto's and $\mathrm{MH}$ ) and random effects (HG and SGS) methods to calculate summary odds ratios (OR) and their 95\% CI. SGS method calculates summary relative risk (RR) instead of OR; however, at low event rates, the distinction is slight and RR can be interpreted as OR [26]. We have presented and compared results of all four methods as summary OR and 95\% CI.

Cochrane recommends Peto's as the least biased and most powerful method when event rates are $<1 \%$, when there is no substantial imbalance between treatment group sizes, and treatment effects are not exceptionally large [29]. Peto's method provides a weighted estimate of the (log) odds ratio under a fixed-effects model [30]. Cochrane recommends $\mathrm{MH}$ without zero-cell corrections for event rates $>1 \%$. Peto and $\mathrm{MH}$ are fixed-effect methods that assume treatment effects to be the same across all study populations, while HG and SGS use randomeffect models incorporating between-study heterogeneity. All analyses were performed in the Metafor package in $\mathrm{R}$ and in SAS.

When one or both treatment arms had no events, some meta-analysis methods could not compute summary estimates. To enable computation, counts were altered in randomly selected studies to allow an exploratory sensitivity analysis. If only one arm had a pooled event count of zero (e.g., 3-0), a study with an observed event was randomly selected, and its zero count changed to one. Changing counts of zero to one in one arm biases towards the null, so our sensitivity analysis is only presented for comparison of statistical methods and not for hypothesis testing.

This study was funded with an unrestricted grant from Novartis Pharmaceuticals. They were not involved in any way in the design, conduct, or interpretation of the results. They were given the opportunity to comment on the draft manuscript in an effort to ensure there were no errors of fact. This article is based on previously conducted studies and does not contain any new studies with human participants or animals performed by any of the authors.

\section{RESULTS}

Database searches identified 12,830 publications, which were assessed for duplication and the inclusion/exclusion criteria by title/abstract review (Fig. 1). Full-text review of 167 publications and excluded 71, leaving 31 anti-IL-17 and 65 anti-TNF trials for inclusion. The included studies are summarized in the Supplementary Material.

Included studies involved 28,209 participants and 33 IBD events. We categorized the 26 axial spondyloarthritis and two peripheral spondyloarthritis trials as 'spondyloarthritis', separate from 'psoriatic arthritis'. Minimal adverse event reporting was found in the main publication, supplementary data, and trials registry for seven anti-TNF trials and one small phase II secukinumab trial, which were excluded (Supplementary Material). Four head-tohead trials comparing anti-IL-17 to anti-TNF and placebo were identified, in total involving two new and two flare IBD events, all in the IL17 arms, with no IBD events in placebo or antiTNF arms [31].

Across trials and interventions, ethnicity and gender were homogenous, while the median RCT period was 16 weeks (Table 1 ).

We found an IBD event description in nine trials to be insufficient in the original publication, supplementary data, and trials registry. In one ixekizumab trial (SPIRIT-P2), and three 


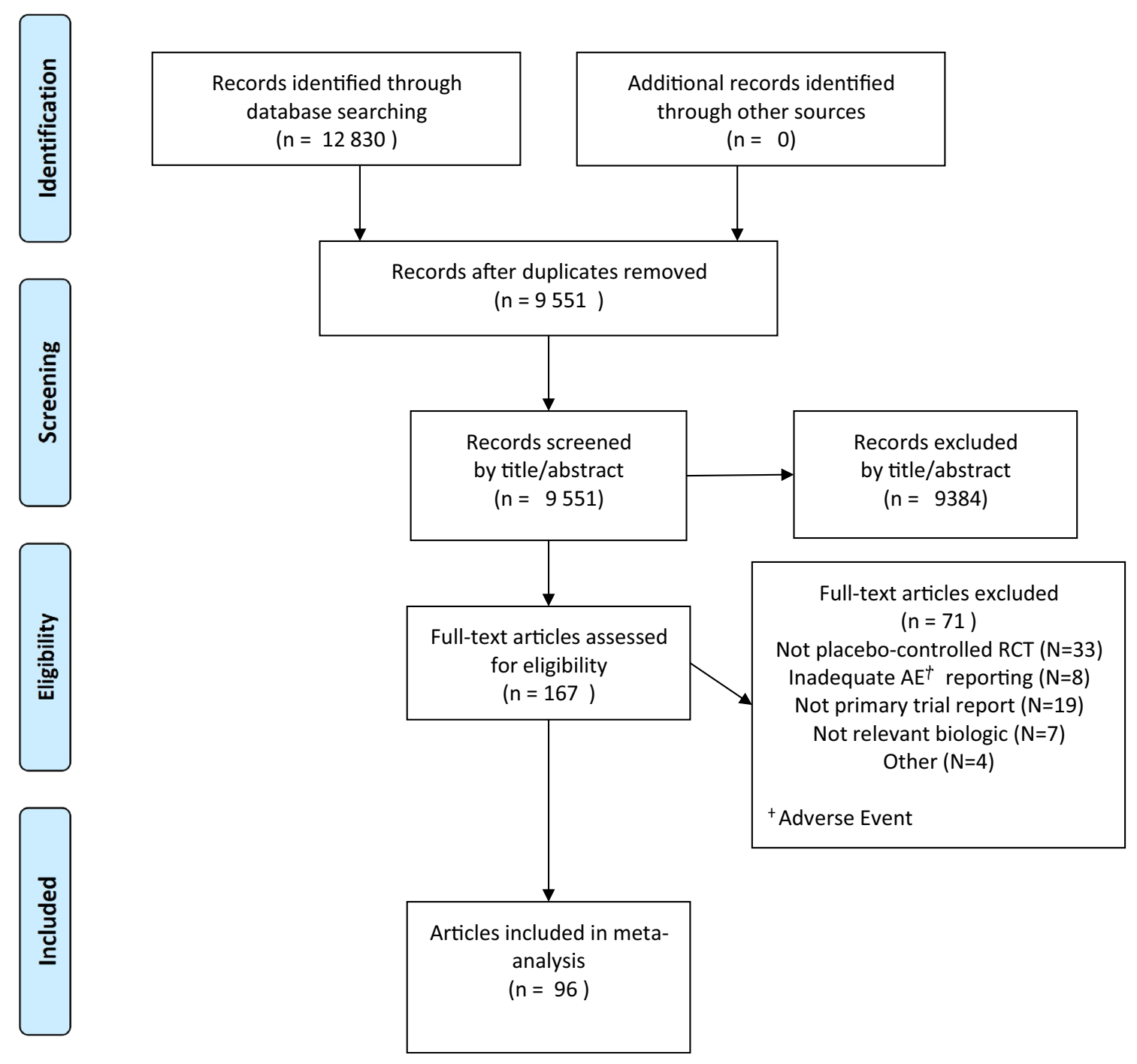

Fig. 1 PRISMA flowchart of study identification process

secukinumab trials (FIXTURE, ERASURE, and NCT03066609), we classified events by contacting the trial sponsor or with a subsequent publication [31-33]. We were unable to classify IBD events in four secukinumab trials (ALLURE, FUTURE-1, FUTURE-2, MEASURE-4) and two anti-TNF trials ([39], ABILITY-3) so assumed these events to be new IBD, adding five new IBD events on secukinumab, four on anti-TNF and two on placebo [34-39].

Active IBD at enrollment was excluded in ten anti-IL-17 and 13 anti-TNF trials. Known IBD was excluded in two trials, both anti-TNF trials for axial spondyloarthritis. Previously diagnosed IBD was reported by only 16 trials, with a pooled prevalence of $3.8 \%$.

IBD events were very rare, occurring in 33 of 28,209 participants, over a median RCT period of 16 weeks. More events occurred in spondyloarthritis cohorts and treatment arms. Twice as many subjects received treatment than placebo. IBD events are listed in the Supplementary Material.

Event rates on placebo and anti-TNF were similar for all conditions, for new and flare IBD events, with no IBD events in anti-TNF trials for psoriasis or PsA. New IBD events on anti-IL17 were rare, occurring with event rates of 0.23 / 
Table 1 Profile of included trial participants, grouped by intervention received

\begin{tabular}{llll}
\hline & $\begin{array}{l}\text { Anti-IL- } \\
\mathbf{1 7}\end{array}$ & $\begin{array}{l}\text { Anti- } \\
\text { TNF }\end{array}$ & Placebo \\
\hline Mean age (years) & 45.7 & 42.9 & 43.8 \\
Male (\%) & 63.2 & 65.1 & 64.5 \\
White/European (\%) & 82.3 & 70.7 & 74.7 \\
$\begin{array}{l}\text { Past IBD excluded by trial } \\
\quad 0\end{array}$ & 3.2 & 2.1 \\
$\quad$ \%) & & & \\
$\begin{array}{l}\text { Active IBD excluded by } \\
\text { trial (\%) }\end{array}$ & 35.5 & 16.9 & 22.9 \\
$\begin{array}{l}\text { Median trial period } \\
\text { (weeks) }\end{array}$ & 16 & 16 & 16 \\
\hline
\end{tabular}

100 patient-years (PY) in psoriasis, 0.61/100 patient years (PY) in psoriatic arthritis and 1.6/ 100 PY in spondyloarthritis. Further rates are listed in the Supplementary Material.

\section{Risk of Bias}

Jadad scores were high with mean $4.2 / 5$ and high agreement $86.2 \%$, Cohen's kappa 0.79 and ICC 0.86 (Supplementary Material). Common reasons for imperfect scores included unevenly sized trial arms and insufficient description of randomization and blinding methods.

\section{Statistical Results}

Meta-analyses pooled all included studies for each condition, comparing new and flare IBD events in treatment and placebo arms in antiTNF, anti-IL-17, ixekizumab, and secukinumab trials. Two-thirds of anti-IL-17 trials and 95\% of anti-TNF trials had no events in either placebo or treatment arms (Table 2). There were only four IBD events in placebo groups, all occurring in spondyloarthritis trials. The lack of events in the placebo arm prevented the use of most meta-analysis methods using observed data, so sensitivity analyses were performed to enable use and comparison of meta-analysis methods.

The low event rate makes heterogeneity difficult to assess, but estimates were very low,

Table 2 Summary of IBD events in RCTs using anti-TNF, secukinumab, or ixekizumab for psoriasis, psoriatic arthritis, or spondyloarthritis

\begin{tabular}{|c|c|c|c|c|c|c|c|c|}
\hline \multirow[t]{2}{*}{ Condition } & \multirow[t]{2}{*}{ Treatment } & \multirow[t]{2}{*}{$\begin{array}{l}\text { Number of } \\
\text { trials }\end{array}$} & \multicolumn{2}{|c|}{$\begin{array}{l}\text { No. of new IBD } \\
\text { cases }\end{array}$} & \multicolumn{2}{|c|}{$\begin{array}{l}\text { No. of flares of } \\
\text { known IBD }\end{array}$} & \multicolumn{2}{|c|}{ Subject count } \\
\hline & & & Treatment & Placebo & Treatment & Placebo & Treatment & Placebo \\
\hline Psoriasis & Secukinumab & 14 & 2 & 0 & 2 & 0 & 3058 & 1345 \\
\hline Psoriasis & Ixekizumab & 3 & 1 & 0 & 1 & 0 & 1653 & 461 \\
\hline Psoriasis & Anti-TNF & 30 & 0 & 0 & 0 & 0 & 6142 & 2788 \\
\hline PsA & Secukinumab & 5 & 4 & 0 & 1 & 0 & 1622 & 760 \\
\hline PsA & Ixekizumab & 2 & 1 & 0 & 0 & 0 & 454 & 224 \\
\hline PsA & Anti-TNF & 14 & 0 & 0 & 0 & 0 & 2242 & 1442 \\
\hline Spondyloarthritis & Secukinumab & 5 & 3 & 0 & 3 & 0 & 801 & 394 \\
\hline Spondyloarthritis & Ixekizumab & 2 & 3 & 0 & 2 & 1 & 376 & 191 \\
\hline Spondyloarthritis & Anti-TNF & 21 & 4 & 3 & 2 & 0 & 2559 & 1697 \\
\hline Total & & 96 & 18 & 3 & 11 & 1 & 18,907 & 9302 \\
\hline
\end{tabular}


with all meta-analyses reporting $I^{2}$ of zero apart from new IBD events in spondyloarthritis antiTNF trials with a low-to-moderate $I^{2}$ of 37.2 (Peto's method). Meta-analysis forest plots have not been provided due to the large range of variation from our analysis of these low and no event rate trials. Instead, forest plots have been used to demonstrate the large uncertainty around estimates of OR for each comparison.

Only one subject in an anti-IL-17 RCT placebo arm had an IBD event, in a spondyloarthritis trial, allowing calculation using all four meta-analysis methods. Comparing one vs. five events, all methods produced similar OR estimates with wide confidence intervals (CI), none finding a significant difference (Table 3).

There were 3-4 new IBD events per arm in spondyloarthritis anti-TNF trials, producing similar effect size and CI estimates by all four meta-analysis methods (Table 4).

Comparisons with zero-count arms were progressed to sensitivity analysis, which produced very wide CI for all analyses. Even the highest observed event count of six produced wide CI upon sensitivity analysis (Fig. 2).

HG and SGS methods were unable to provide an estimate of effect and/or CI when event rates were $<3$ in both intervention arms and only a single study reported any events. For all comparisons not involving zero, $\mathrm{MH}$ calculated results similar to those from $\mathrm{HG}$ and SGS methods.

\section{DISCUSSION}

IBD events on anti-IL-17 therapy and placebo were very rare, with no demonstrable difference in event rate. Our analyses were severely limited by low event rates and the performance of metaanalysis methods on this dataset. This study is the largest meta-analysis of anti-IL-17 and antiTNF trials and aimed to limit reporting bias and to assess the use of meta-analysis techniques recommended for low event rates. Previous studies have mostly been per-medication integrated analyses that pool all available data at the patient level, without performing metaanalysis looking for an effect.
Despite pooling 31 anti-IL-17 RCTs, our comparisons were hindered by event scarcity and counts of zero. We chose to pool biologics in the same class but not different diseases and not new and flare events. Events were more common in both anti-TNF or anti-IL-17 than placebo, with new IBD events twice as common as flares on treatment or placebo, despite an estimated $3.8 \%$ of participants reporting past IBD.

For a clinically relevant comparison to the anti-IL-17 analysis, we also performed a metaanalysis of IBD events in anti-TNF trials. AntiTNF agents are the major alternative to anti-IL17 for inflammatory arthritis and psoriasis, and can also produce adverse effects. After our literature search, two more head-to-head trials were published, comparing adalimumab to antiIL-17 agents-one against secukinumab and one against ixekizumab $[40,41]$. Each trial had two IBD events on anti-IL-17 treatment and none on adalimumab, rates likely insufficient for hypothesis testing [40, 41]. Additionally, head-to-head comparisons may be confounded by an IBD-suppressing effect of anti-TNF agents given their use as a licensed treatment for IBD. Our pooled event counts did not exhibit any IBD-suppressing effect of anti-TNF, with twice as many IBD events on anti-TNF than placebo, although events were of very low frequency. Meta-analysis of events on anti-TNF in spondyloarthritis produced an OR with wide CI crossing 1. The comparison of IBD events on anti-TNF requires many more events, and is of interest given the known phenomenon of paradoxical effects. Our findings are insufficient to compare event rates on anti-TNF to anti-IL17 , and reiterate how at low event rates, recognized therapeutic effects may not be evident.

We reported a similar frequency of IBD events to previous cohorts, which have observed IBD event rates of 0.1-0.2 /100 PY in psoriasis, $0.16-0.57 / 100 \mathrm{PY}$ in psoriatic arthritis, and 1-2/100 PY in spondyloarthritis patients on biologic therapy $[19,42-45]$. Reporting bias may have increased the new IBD event count, as many RCTs consist of biologic-naive subjects who may not have previously been regularly asked about bowel symptoms. 


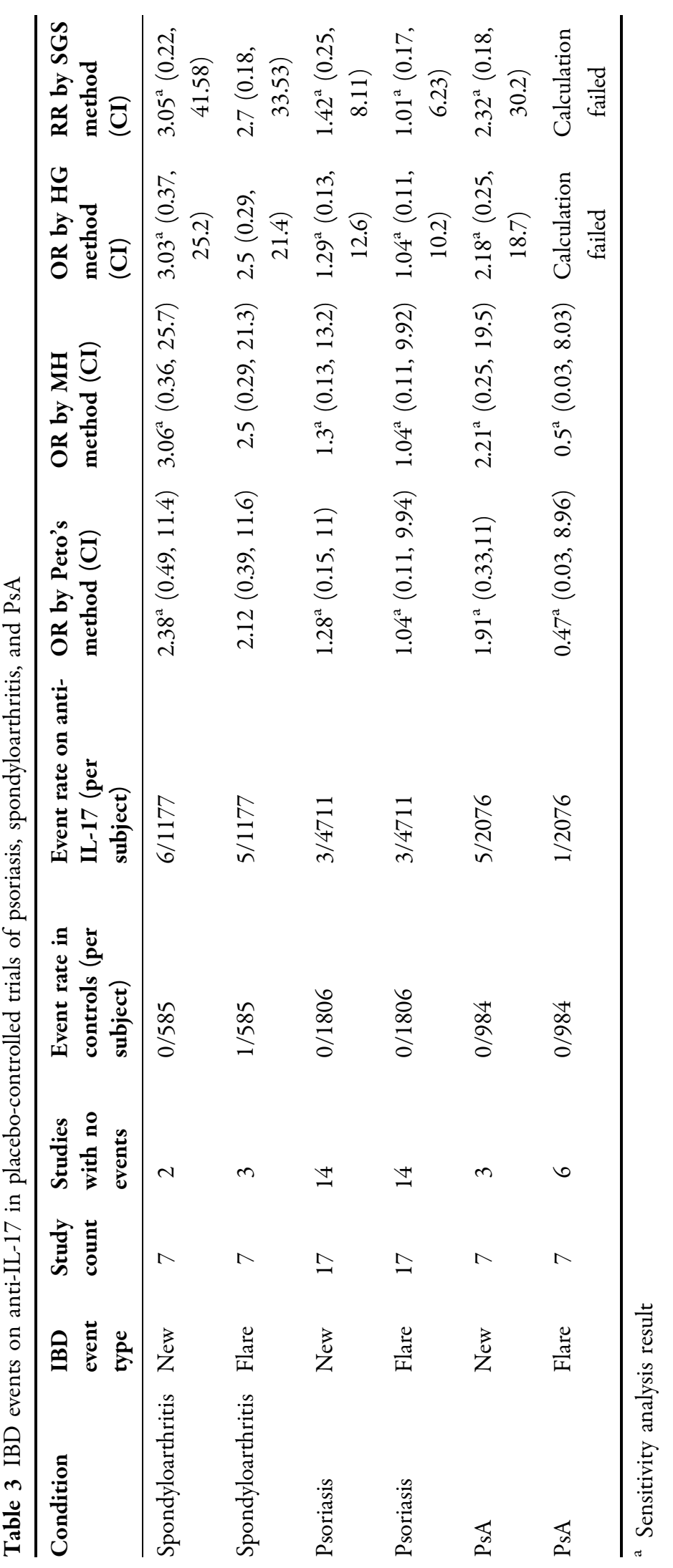


Table 4 IBD events on anti-TNF in placebo-controlled trials of psoriasis, spondyloarthritis, and PsA

\begin{tabular}{|c|c|c|c|c|c|c|c|c|c|}
\hline Condition & $\begin{array}{l}\text { IBD } \\
\text { event } \\
\text { type }\end{array}$ & $\begin{array}{l}\text { Study } \\
\text { count }\end{array}$ & $\begin{array}{l}\text { Studies } \\
\text { with no } \\
\text { events }\end{array}$ & $\begin{array}{l}\text { Event } \\
\text { rate in } \\
\text { controls } \\
\text { (per } \\
\text { subject) }\end{array}$ & $\begin{array}{l}\text { Event } \\
\text { rate on } \\
\text { anti-TNF } \\
\text { (per } \\
\text { subject) }\end{array}$ & $\begin{array}{l}\text { OR by } \\
\text { Peto's } \\
\text { method } \\
(\mathrm{CI})\end{array}$ & $\begin{array}{l}\text { OR by } \\
\text { MH } \\
\text { method } \\
\text { (CI) }\end{array}$ & $\begin{array}{l}\text { OR by HG } \\
\text { method } \\
(\mathrm{CI})\end{array}$ & $\begin{array}{l}\text { RR by SGS } \\
\text { method } \\
\text { (CI) }\end{array}$ \\
\hline Spondyloarthritis & New & 21 & 18 & $3 / 1697$ & $4 / 2559$ & $\begin{array}{l}1.22 \\
\quad(0.27 \\
5.48)\end{array}$ & $\begin{array}{l}1.21 \\
(0.28 \\
5.22)\end{array}$ & $\begin{array}{c}1.22(0.27 \\
5.51)\end{array}$ & $\begin{array}{c}1.14(0.37 \\
3.58)\end{array}$ \\
\hline Spondyloarthritis & Flare & 21 & 20 & $0 / 1697$ & $2 / 2559$ & $\begin{array}{l}1.04^{\mathrm{a}} \\
(0.09 \\
11.4)\end{array}$ & $\begin{array}{c}1.04^{\mathrm{a}} \\
(0.09 \\
11.6)\end{array}$ & $\begin{array}{l}\text { Calculation } \\
\text { failed }\end{array}$ & $\begin{array}{l}\text { Calculation } \\
\text { failed }\end{array}$ \\
\hline Psoriasis & New & 30 & 30 & $0 / 2788$ & $0 / 6142$ & $\mathrm{~N} / \mathrm{A}$ & $\mathrm{N} / \mathrm{A}$ & $\mathrm{N} / \mathrm{A}$ & N/A \\
\hline Psoriasis & Flare & 30 & 30 & $0 / 2788$ & $0 / 6142$ & $\mathrm{~N} / \mathrm{A}$ & N/A & $\mathrm{N} / \mathrm{A}$ & $\mathrm{N} / \mathrm{A}$ \\
\hline PsA & New & 14 & 14 & $0 / 1442$ & $0 / 2242$ & $\mathrm{~N} / \mathrm{A}$ & N/A & $\mathrm{N} / \mathrm{A}$ & N/A \\
\hline PsA & Flare & 14 & 14 & $0 / 1442$ & $0 / 2242$ & $\mathrm{~N} / \mathrm{A}$ & $\mathrm{N} / \mathrm{A}$ & $\mathrm{N} / \mathrm{A}$ & $\mathrm{N} / \mathrm{A}$ \\
\hline
\end{tabular}

${ }^{a}$ Sensitivity analysis result. Sensitivity analysis was not performed if no events occurred in either arm

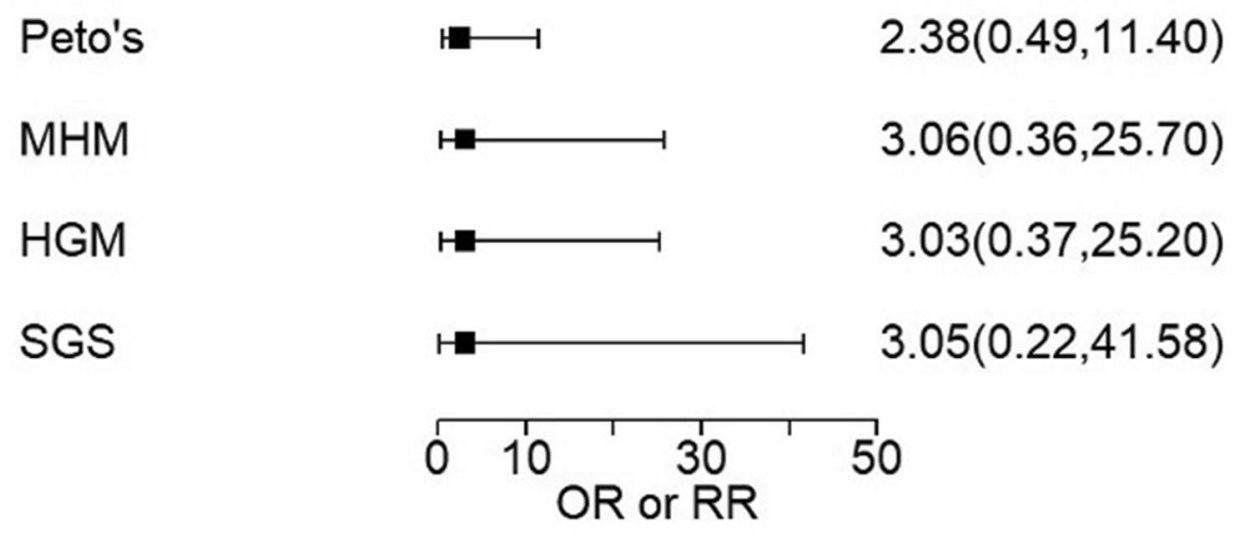

Fig. 2 Sensitivity meta-analysis of new IBD in anti-IL-17 spondyloarthritis trials, comparing event rates of $1 / 585$ to $6 / 1177$

Most RCTs had zero events in one or both arms, suggesting that event frequency was low but providing limited information for OR/RR calculation. We are not aware of any statistical method that can obtain pooled estimates when both intervention arms have no events. Sensitivity analyses enabled us to compare the utility of four meta-analysis methods recommended for low event rate data.
Previous publications report similar IBD event rates on anti-IL-17, and also faced difficulties from rare events, pooling multiple diseases and from choosing a statistical method where no stand-out exists. Previous integrated analyses have pooled patient-level data from ixekizumab or secukinumab studies of psoriasis, psoriatic arthritis and axial spondyloarthritis, and report CI but no formal hypothesis testing. This would require accurate modeling of 
between-disease and between-study variability [46].

Two previous meta-analyses of anti-IL-17 trials for arthritis and psoriasis identified fewer IBD events than our study, with one study performing meta-analysis with zero pooled events on placebo $[21,22,47]$. These metaanalyses pooled quite different studies, including blinded and open-label periods from trials of rheumatoid arthritis, psoriasis, psoriatic arthritis, and axial spondyloarthritis. Their inclusive approach marginally increased event counts at the expense of increasing betweenstudy heterogeneity. Both previous meta-analyses chose a $\mathrm{MH}$ risk difference method, which is limited by low power and an inability to process pooled counts of zero $[29,30]$. Authors addressing this topic face statistical compromises and benefit from discussion of the statistical challenges faced by each subsequent publication.

A recently published French study of 16,793 new users of anti-IL-17 agents did not find an increased risk of IBD compared to new users of etanercept [48]. This study design was able to control for severity of underlying disease, which could potentially affect the rate of new IBD.

Our novel approach involved meta-analysis using multiple methods, with the a priori plan to perform sensitivity analyses, an approach suggested by previous authors [30, 47]. Our analyses of observed data and sensitivity analyses produced point estimates with large degrees of uncertainty, inadequate for confident hypothesis testing in this case.

Increased sample size without more events did not improve certainty using either Peto's or $\mathrm{MH}$ methods. This occurs because these two methods exclude 0-0 studies from analysis $[47,49]$. Future studies using these methods should capture more events, ideally more than three per arm. While $0-0$ trials are not informative for RR calculation, they are evidence for no difference in absolute risk, which in this case is more clinically relevant than RR. Quantification of absolute and relative risk of treatmentinduced IBD is of great importance as spondyloarthritis has few treatment options and IBD flares can have substantial morbidity.
The four meta-analysis methods demonstrated lower certainty at lower event rates. With sufficient events, all methods provided similar OR estimates, although CI were wide, with Peto's method often producing the narrowest CI. Peto's major limitation for biologic RCTs is that it underestimates event rates in the larger arm when intervention groups are of unequal size, and most trials we included had intervention arms twice as large as the control. Peto's and MH are fixed-effect methods, less suitable when between-study variation is significant. In this case, we did not pool different diseases and calculated measures of heterogeneity did not indicate significant heterogeneity. SGS and HG use random-effects but on multiple analyses failed to calculate any estimate when event counts were $<3$ and when only one study reported any events. The limitations of each method reiterate the recommendations of previous authors who state that meta-analysis method choice is critical and should be accompanied by sensitivity analysis using multiple methods when event data are sparse [30].

We included studies of variable design, including duration, follow-up protocols, and reporting. All were biologic RCTs, so the studies were likely as similar as possible for a metaanalysis. We were unable to account for duration in our meta-analysis but two-thirds of RCTs were of $12-16$ weeks long. Use of only doubleblinded periods aimed to reduce reporting bias but excluded prolonged open-label follow-up studies.

Reporting of IBD events by authors was variable, with no standard definition of an IBDrelated adverse event, and few studies reported pre-existing IBD. Some events, such as single, self-limited episodes of diarrhea, were described but not investigated, and trial protocols regarding possible IBD have changed over time. Eleven IBD events were inadequately described (five secukinumab, four anti-TNF, two placebo), so we assumed they were new IBD events, introducing slight bias towards new IBD. Exclusion criteria also varied between RCTs and were at times vague, e.g., 'systemic inflammatory conditions that may confound evaluation of benefit'. Once a possible safety signal is 
identified in a drug class, particularly one with a novel mechanism of action, enhanced systematic reporting of that adverse event would help to better elucidate the nature of any risk, and reassure prescribers and regulators. In the case of IL-17A inhibitors, understanding this risk would be more achievable with standardization. This issue would benefit from standardization of IBD event reporting, including an unequivocal IBD event rate, investigation of suspected IBD events, and reporting of previous IBD in participants.

IBD risk from treatments is ideally assessed by large, prolonged placebo-controlled RCT data. Head-to-head comparisons of biologics are confounded by recognized IBD suppressive effects. In the absence of an ideal dataset, another confounded but potentially helpful approach would be to observe IBD events in a biologics registry or long-term cohort. High-risk cohorts of individuals with spondyloarthritis with a past or family history of IBD could be regularly surveyed after commencing treatment.

The limitations of the current study are low event rates, inconsistent standard definition of an IBD-related adverse event, and variable reporting of the detail of IBD event rates.

\section{CONCLUSIONS}

Our systematic review and meta-analysis of anti-IL-17 and anti-TNF RCTs found new and flare IBD events to be rare on these treatments and rarer on placebo. We compared four methods of meta-analysis, which provided similar point estimates but wide confidence intervals, and we were not able to perform confident hypothesis testing. Many of our comparisons had pooled event rates of zero, preventing the use of most meta-analysis methods using observed data. None of our meta-analyses of observed data or sensitivity analyses demonstrated a significant difference in event rate between anti-IL-17 agents or antiTNF and placebo.

Our statistical findings reiterate those of previous studies, finding limitations of all methods tested. Requirements for improved hypothesis testing include more IBD events (even if the rate is lowered), more events in placebo arms and equal treatment arm sizes if using Peto's method. Informative IBD event data might be found from high-risk groups in placebo-controlled RCTs or from observational data.

\section{ACKNOWLEDGEMENTS}

Funding. This work was supported by an unrestricted grant from Novartis Pharmaceuticals Australia. This funding paid for investigator time, statistical analysis, and the journal's Rapid Service Fee.

Authorship. All named authors meet the International Committee of Medical Journal Editors (ICMJE) criteria for authorship for this article, take responsibility for the integrity of the work as a whole, and have given their approval for this version to be published.

Author Contributions. Concept and design: SLT, JC, DFL, DR, GRS, PCR. Statistical analysis, SFZ and EGR. Drafting the manuscript: All authors. Approving the final version for publication: All authors.

Disclosures. Jasmine Chin, David FL Liew, Syeda Farah Zahir, Elizabeth G Ryan, Diana Rubel and Steven L Truong have nothing to disclose. Graham Radford-Smith reports advisory board fees from Janssen, Takeda, Novartis, Pfizer, AbbVie, Ferring; speakers fees from Pfizer, Takeda; research grants from Janssen, Takeda. Philip C Robinson reports personal fees from AbbVie, Atom Biosciences, Eli Lilly, Gilead, Janssen, Novartis, Pfizer, Roche, UCB Pharma; research grants from Janssen, Pfizer, Novartis and UCB Pharma; meeting attendance support from BMS, Lilly, and UCB all outside the submitted work.

Compliance with Ethical Guidelines. This article is based on previously conducted studies and does not contain any new studies with 
human participants or animals performed by any of the authors.

Data Availability. All data generated or analyzed during this study are included in this published article/as supplementary information files.

Open Access. This article is licensed under a Creative Commons Attribution-NonCommercial 4.0 International License, which permits any non-commercial use, sharing, adaptation, distribution and reproduction in any medium or format, as long as you give appropriate credit to the original author(s) and the source, provide a link to the Creative Commons licence, and indicate if changes were made. The images or other third party material in this article are included in the article's Creative Commons licence, unless indicated otherwise in a credit line to the material. If material is not included in the article's Creative Commons licence and your intended use is not permitted by statutory regulation or exceeds the permitted use, you will need to obtain permission directly from the copyright holder. To view a copy of this licence, visit http://creativecommons.org/licenses/by$\mathrm{nc} / 4.0 /$.

\section{REFERENCES}

1. Robinson PC, van der Linden S, Khan MA, Taylor WJ. Axial spondyloarthritis: concept, construct, classification and implications for therapy. Nat Rev Rheumatol. 2020;17(2):109-18.

2. Robinson PC, Sengupta R, Siebert S. Non-radiographic axial spondyloarthritis (nr-axSpA): advances in classification, imaging and therapy. Rheumatol Ther. 2019;6(2):165-77.

3. Siebert S, Millar NL, McInnes IB. Why did IL-23p19 inhibition fail in AS: a tale of tissues, trials or translation? Ann Rheum Dis. 2019;78:1015-8.

4. Moschen AR, Tilg H, Raine T. IL-12, IL-23 and IL-17 in IBD: immunobiology and therapeutic targeting. Nat Rev Gastroenterol Hepatol. 2019;16(3):185-96.

5. Hovhannisyan Z, Treatman J, Littman DR, Mayer L. Characterization of interleukin-17-producing regulatory $\mathrm{T}$ cells in inflamed intestinal mucosa from patients with inflammatory bowel diseases. Gastroenterology. 2011;140(3):957-65.

6. Ueno A, Jijon H, Chan R, Ford K, Hirota C, Kaplan $\mathrm{GG}$, et al. Increased prevalence of circulating novel IL-17 secreting Foxp3 expressing $\mathrm{CD}^{4+} \mathrm{T}$ cells and defective suppressive function of circulating Foxp ${ }^{3+}$ regulatory cells support plasticity between Th17 and regulatory $\mathrm{T}$ cells in inflammatory bowel disease patients. Inflamm Bowel Dis. 2013;19(12): 2522-34.

7. Chong WP, Mattapallil MJ, Raychaudhuri K, Bing SJ, Wu S, Zhong Y, et al. The Cytokine IL-17A Limits Th17 Pathogenicity via a Negative Feedback Loop Driven by Autocrine Induction of IL-24. Immunity. 2020;53(2):384-97 (e5).

8. Targan SR, Feagan B, Vermeire S, Panaccione R, Melmed GY, Landers C, et al. A randomized, double-blind, placebo-controlled phase 2 study of brodalumab in patients with moderate-to-severe Crohn's disease. Am J Gastroenterol. 2016;111(11): 1599-607.

9. Hueber W, Sands BE, Lewitzky S, Vandemeulebroecke M, Reinisch W, Higgins PD, et al. Secukinumab, a human anti-IL-17A monoclonal antibody, for moderate to severe Crohn's disease: unexpected results of a randomised, double-blind placebo-controlled trial. Gut. 2012;61(12): 1693-700.

10. Fujino S, Andoh A, Bamba S, Ogawa A, Hata K, Araki $\mathrm{Y}$, et al. Increased expression of interleukin 17 in inflammatory bowel disease. Gut. 2003;52(1): 65-70.

11. Cullen G, Kroshinsky D, Cheifetz AS, Korzenik JR. Psoriasis associated with anti-tumour necrosis factor therapy in inflammatory bowel disease: a new series and a review of 120 cases from the literature. Aliment Pharmacol Ther. 2011;34(11-12):1318-27.

12. Fouache D, Goeb V, Massy-Guillemant N, Avenel G, Bacquet-Deschryver $\mathrm{H}$, Kozyreff-Meurice $\mathrm{M}$, et al. Paradoxical adverse events of anti-tumour necrosis factor therapy for spondyloarthropathies: a retrospective study. Rheumatology (Oxford). 2009;48(7): 761-4.

13. Seror R, Richez C, Sordet C, Rist S, Gossec L, Direz $\mathrm{G}$, et al. Pattern of demyelination occurring during anti-TNF-alpha therapy: a French national survey. Rheumatology (Oxford). 2013;52(5):868-74.

14. Charlton R, Green A, Shaddick G, Snowball J, Nightingale A, Tillett $W$, et al. Risk of uveitis and inflammatory bowel disease in people with psoriatic arthritis: a population-based cohort study. Ann Rheum Dis. 2018;77(2):277-80. 
15. Orrell KA, Murphrey M, Kelm RC, Lee HH, Pease $\mathrm{DR}$, Laumann AE, et al. Inflammatory bowel disease events after exposure to interleukin 17 inhibitors secukinumab and ixekizumab: postmarketing analysis from the RADAR ("research on adverse drug events and reports") program. J Am Acad Dermatol. 2018;79(4):777-8.

16. Schreiber S, Colombel JF, Feagan BG, Reich K, Deodhar AA, McInnes IB, et al. Incidence rates of inflammatory bowel disease in patients with psoriasis, psoriatic arthritis and ankylosing spondylitis treated with secukinumab: a retrospective analysis of pooled data from 21 clinical trials. Ann Rheum Dis. 2019;78(4):473-9.

17. Mease P, Roussou E, Burmester GR, Goupille P, Gottlieb A, Moriarty SR, et al. Safety of ixekizumab in patients with psoriatic arthritis: results from a pooled analysis of three clinical trials. Arthritis Care Res (Hoboken). 2019;71(3):367-78.

18. Reich K, Leonardi C, Langley RG, Warren RB, Bachelez H, Romiti R, et al. Inflammatory bowel disease among patients with psoriasis treated with ixekizumab: a presentation of adjudicated data from an integrated database of 7 randomized controlled and uncontrolled trials. J Am Acad Dermatol. 2017;76(3):441-8 (e2).

19. Genovese MC, Mysler E, Tomita T, Papp KA, Salvarani C, Schwartzman S, et al. Safety of ixekizumab in adult patients with plaque psoriasis, psoriatic arthritis and axial spondyloarthritis: data from 21 clinical trials. Rheumatology (Oxford). 2020;59(12): 3834-44.

20. Armstrong A, Paul C, Puig L, Boehncke WH, Freeman M, Torii H, et al. Safety of ixekizumab treatment for up to 5 years in adult patients with moderate-to-severe psoriasis: results from greater than 17,000 patient-years of exposure. Dermatol Ther (Heidelb). 2020;10(1):133-50.

21. Burisch J, Eigner W, Schreiber S, Aletaha D, Weninger $\mathrm{W}$, Trauner $\mathrm{M}$, et al. Risk for development of inflammatory bowel disease under inhibition of interleukin 17: a systematic review and meta-analysis. PLoS ONE. 2020;15(5): e0233781.

22. Yamada A, Wang J, Komaki Y, Komaki F, Micic D, Sakuraba A. Systematic review with meta-analysis: risk of new onset IBD with the use of anti-interleukin-17 agents. Aliment Pharmacol Ther. 2019;50(4):373-85.

23. Jadad AR, Moore RA, Carroll D, Jenkinson C, Reynolds DJ, Gavaghan DJ, et al. Assessing the quality of reports of randomized clinical trials: is blinding necessary? Control Clin Trials. 1996;17(1):1-12.
24. Carron P, Varkas G, Cypers H, Van Praet L, Elewaut D, Van den Bosch F, et al. Anti-TNF-induced remission in very early peripheral spondyloarthritis: the CRESPA study. Ann Rheum Dis. 2017;76(8): 1389-95.

25. Yusuf S, Peto R, Lewis J, Collins R, Sleight P. Beta blockade during and after myocardial infarction: an overview of the randomized trials. Prog Cardiovasc Dis. $1985 ; 27(5): 335-71$.

26. Shuster JJ, Walker MA. Low-event-rate meta-analyses of clinical trials: implementing good practices. Stat Med. 2016;35(14):2467-78.

27. Shuster JJ, Guo JD, Skyler JS. Meta-analysis of safety for low event-rate binomial trials. Res Synth Methods. 2012;3(1):30-50.

28. Stijnen T, Hamza TH, Ozdemir P. Random effects meta-analysis of event outcome in the framework of the generalized linear mixed model with applications in sparse data. Stat Med. 2010;29(29): 3046-67.

29. Higgins JPT, Thomas J, Chandler J, Cumpston M, Li T, Page M. Cochrane handbook for systematic reviews of interventions. Hoboken: Wiley; 2019.

30. Bradburn MJ, Deeks JJ, Berlin JA, Russell LA. Much ado about nothing: a comparison of the performance of meta-analytical methods with rare events. Stat Med. 2007;26(1):53-77.

31. Cai L, Zhang JZ, Yao X, Gu J, Liu QZ, Zheng M, et al. Secukinumab demonstrates high efficacy and a favorable safety profile over 52 weeks in Chinese patients with moderate to severe plaque psoriasis. Chin Med J (Engl). 2020;133(22):2665-73.

32. Nash P, Kirkham B, Okada M, Rahman P, Combe B, Burmester GR, et al. Ixekizumab for the treatment of patients with active psoriatic arthritis and an inadequate response to tumour necrosis factor inhibitors: results from the 24-week randomised, double-blind, placebo-controlled period of the SPIRIT-P2 phase 3 trial. Lancet. 2017;389(10086): 2317-27.

33. Langley RG, Elewski BE, Lebwohl M, Reich K, Griffiths CE, Papp K, et al. Secukinumab in plaque psoriasis-results of two phase 3 trials. N Engl J Med. 2014;371(4):326-38.

34. Elewski BE, Baddley JW, Deodhar AA, Magrey M, Rich PA, Soriano ER, et al. Association of secukinumab treatment with tuberculosis reactivation in patients with psoriasis, psoriatic arthritis, or ankylosing spondylitis. JAMA Dermatol. 2021;157(1): 43-51. 
35. Mease PJ, McInnes IB, Kirkham B, Kavanaugh A, Rahman P, van der Heijde D, et al. Secukinumab inhibition of interleukin-17A in patients with psoriatic arthritis. N Engl J Med. 2015;373(14): 1329-39.

36. McInnes IB, Mease PJ, Kirkham B, Kavanaugh A, Ritchlin CT, Rahman P, et al. Secukinumab, a human anti-interleukin-17A monoclonal antibody, in patients with psoriatic arthritis (FUTURE 2): a randomised, double-blind, placebo-controlled, phase 3 trial. Lancet. 2015;386(9999):1137-46.

37. Kivitz AJ, Wagner U, Dokoupilova E, Supronik J, Martin R, Talloczy Z, et al. Efficacy and safety of secukinumab $150 \mathrm{mg}$ with and without loading regimen in ankylosing spondylitis: 104-week results from MEASURE 4 study. Rheumatol Ther. 2018;5(2):447-62.

38. Landewe R, Sieper J, Mease P, Inman RD, Lambert RG, Deodhar A, et al. Efficacy and safety of continuing versus withdrawing adalimumab therapy in maintaining remission in patients with non-radiographic axial spondyloarthritis (ABILITY-3): a multicentre, randomised, double-blind study. Lancet. 2018;392(10142):134-44.

39. Davis JC Jr, Van Der Heijde D, Braun J, Dougados M, Cush J, Clegg DO, et al. Recombinant human tumor necrosis factor receptor (etanercept) for treating ankylosing spondylitis: a randomized, controlled trial. Arthritis Rheum. 2003;48(11): 3230-6.

40. Mease PJ, Smolen JS, Behrens F, Nash P, Liu Leage S, $\mathrm{Li}$ L, et al. A head-to-head comparison of the efficacy and safety of ixekizumab and adalimumab in biological-naive patients with active psoriatic arthritis: 24-week results of a randomised, open-label, blinded-assessor trial. Ann Rheum Dis. 2020;79(1):123-31.

41. McInnes IB, Behrens F, Mease PJ, Kavanaugh A, Ritchlin C, Nash P, et al. Secukinumab versus adalimumab for treatment of active psoriatic arthritis (EXCEED): a double-blind, parallel-group, randomised, active-controlled, phase $3 \mathrm{~b}$ trial. Lancet. 2020;395(10235):1496-505.
42. Egeberg A, Thyssen JP, Burisch J, Colombel JF. Incidence and risk of inflammatory bowel disease in patients with psoriasis-a nationwide 20-year cohort study. J Invest Dermatol. 2019;139(2):316-23.

43. Egeberg A, Mallbris L, Warren RB, Bachelez H, Gislason GH, Hansen PR, et al. Association between psoriasis and inflammatory bowel disease: a Danish nationwide cohort study. $\mathrm{Br} \mathrm{J}$ Dermatol. 2016;175(3):487-92.

44. Kavanaugh A, Papp K, Gottlieb AB, de Jong E, Chakravarty SD, Kafka S, et al. Demography, baseline disease characteristics, and treatment history of psoriasis patients with self-reported psoriatic arthritis enrolled in the PSOLAR registry. BMC Rheumatol. 2018;2:29.

45. Walsh JA, Song X, Kim G, Park Y. Evaluation of the comorbidity burden in patients with ankylosing spondylitis treated with tumour necrosis factor inhibitors using a large administrative claims data set. J Pharm Health Serv Res. 2018;9(2):115-21.

46. Hussong AM, Curran PJ, Bauer DJ. Integrative data analysis in clinical psychology research. Annu Rev Clin Psychol. 2013;9:61-89.

47. Sweeting MJ, Sutton AJ, Lambert PC. What to add to nothing? Use and avoidance of continuity corrections in meta-analysis of sparse data. Stat Med. 2004;23(9):1351-75.

48. Penso L, Bergqvist C, Meyer A, Herlemont P, Weill A, Zureik M, et al. Risk of inflammatory bowel disease in patients with psoriasis, psoriatic arthritis and ankylosing spondylitis initiating interleukin 17 inhibitors: a nationwide population-based study using the French national health data system. Arthritis Rheumatol. 2021. https://doi.org/10.1002/ art.41923.

49. Efthimiou O. Practical guide to the meta-analysis of rare events. Evid Based Ment Health. 2018;21(2): 72-6. 